\title{
Gli effetti della subgravità e i metodi per riprodurla a terra e in volo $(*)$
}

\author{
(x. MeIner! (*k)
}

Ricevuto il 19 Febbraio 1963

\begin{abstract}
Riassunto. - L’A. enuncia brevemente il problema della subgravità descrivendo le principali soluzioni sperimentali escogitate nel corso dell'ultimo decennio per rispondere ai vari interrogativi che tale particolare caratteristica del volo spaziale, clie non trova riscontro in alcuna altra condizione nota all'uomo, pone agli studiosi, biologi e tecnici.

L'A. passa quindi a descrivere i principali metodi e dispositivi per riprodurre lo stato di subgravità, distinguendo i sistemi " a terra ", auspicabili per la loro agevolezza, sicurezza, ma che in genere non riproducono che taluni aspetti fisiopsicologici dello stato subgravitazionale, dai sistemi, ben più indaginosi, che consentono di realizzare stati di subgravità reali e completi (voli parabolici, lanei suborbitali e orbitali di missili). Passa infine a esporre quanto è stato fatto in questo campo presso il Centro di Studi e Ricerche di Medicina Aeronautica e Spaziale di Roma, impiegando la "Torre di Subgravità", ideata e costruita presso detto Centro, la quale consente di realizzare veri stati di subgravità, sia pure di breve durata, preceduti e seguiti da accelerazioni. La "Torre di Subgravità " ha permesso di affrontare aleuni problemi inerenti al volo spaziale, soprattutto quelli posti dagli stati di transizione tra le fasi attive e quella passiva del volo, proponendo solu. zioni di non trascurabile interesse pratico (comportamento psicomotorio dei soggetti, ruolo del labirinto in toto e delle sue singole componenti, ecc.).

I'A. conclude quindi facendo rilevare l'interesse di questa metodica, suscettibile di sviluppi anche più ampi, che può ben allinearsi nel campo mondiale alle ricerche inerenti al volo spaziale.
\end{abstract}

(*) Nota presentata al $2 \circ$ Congresso Internazionale Tecnico Scientifico dello Spazio. Roma, 19-23 Giugno 1962.

(**) Ispettorato di Sanità Aeronautica. Capo dell'Ispettorato. Ten. Gen. Medico C.S.A. Prof. Dott. Isomonaco.

Centro di studi e Ricerche di Medicina Aeronantica e Spaziale. Direttore: Ten. Col. Medico C.S.A. Prof. Dott. A. Scano. 
Sc uMARY. ...- The $A$. briefly enunciates the problem of subgravity, toge ther with an account of the more creditable experimental solutions devised in the last ten years with respect to the problems posed to scientists, biologists and expert engineers by this peculiar and exclusive feature of space flight.

The Author then describes the chief methods and physical means used to simulate subgravity conditions: a distinction is made, in the first place, between "ground" methods (the use of which is recommended for reasons of easy performance and greater security, while noting, however, that they usually permit to investigate only a few of the psychophysiological aspects occurring in space flight) and the nuch more cumbersome methods througl which real and complete subgravity conditions can be at.taned (parabolic flight, suborbital and orbital launching of missiles). Thereafter he reports on the accomplishments and activities of the Center of Studies and Researches of derospace Medicine in Rome by means of the Subgravity Tower designed and installed at the Center's own initiative.

This 'Tower permits to obtain real, though short lasting subgravity conditions, preceded and followed by periods of acceleration. Using the subgravity Tower it has been possible to tackle a few problens of spaceflight, particularly in respect of the transition between the active and passive stages of space flight, and solutions ofsignificant practical consequences could be put forward (psycholmotorial behaviour of subjects; the role played by the labyrinth as a whole and by its individual components, etc.).

In conclusion the Author emphasizes the interest presented by rhese methods, and the possibility of futhering them on a larger scale, within the scope of world researches on space-flight.

Fin da quando l'nomo si affaceiò per la prima rolta, sia pure soltanto con lo sguardo della mente, sullo spazio cosmico, a lui si presento il problema di come l'organismo a la psiche umani arrebbero potuto reagire a quella condizione, quasi inconcepibile per la nostra mentalità legata alla Terra che è la subgravità, in cui le più elementari basi dell'orientamento, le nozioni di alto e basso, di orizzontale e rerticale, ressano di avere un senso oggettivo per trasformarsi in riferimenti puramente soggettivi e instabili.

Ma da quando il volo cosmieo è diventato una realtà imminente, oggi in parte già in via di attuazione, la necessità di conoscere sperimentalmente il comportamento dell'uomo in subgravita si è fatta pressante. Gauer et. al, in tempi relativamente lontani, Campbell (1951) alla vigilia delle prime realizzazioni spaziali, e alt ì ancora avevano puntualizzato il problema, cereando di estrapolare, sulla base di cognizioni note, gli effetti della subgravita, giustamente supposta capace di influire grandemente sulla funzione labirintica, soprattutto su quella utriculo-saceulare, essendo l'apparato in questione il più specificamente e direttamente ricettivo agli effetti della gravità. 
Tra i molti studi preliminari giova rammentare quelli di Haber e Haber, che nel 1951 descrissero la possibilità di eseguire ricerche di ordine fisiologico su soggetti trovantisi in stato di subgravita entro l'abitacolo di aerei percorrenti segmenti di traiettorie balistiche kepleriane. Si tratta di una tecnica che ha dato in seguito promettenti risultati sperimentali.

A questo punto conviene ricordare che tutte le metodiche ideate per indurre stati di subgravità - cioè di riduzione più o meno accentuata della normale gravità terrestre fino anche all'annullamento di essa (gravità zero) - raggiungono il loro scopo in modo dinamico, contrapponendo alla forza di gravità un'altra forza uguale, o quasi uguale, e contraria. Oltre a questo esiste uno stato di vera assenza di gravità, per noi ancora irrealizzabile, che potremmo definire subgravità "statica " (Armstrong, 1960), che si ha nello spazio in punti sufficientemente lontani da qualsiasi corpo celeste perché il loro campo gravitazionale sia praticamente privo di efficacia. Per esempio a $58.000 \mathrm{~km}$ dalla Terra l'accelerazione di gravità è ridotta a solo $0,01 \mathrm{G}$.

Circa dagli inizi della seconda metà di questo secolo fino ad oggi le ricerche intese a studiare gli effetti psicologici della subgravità, a dirimere le prime questioni teoricamente proposte e a rispondere agli interrogativi suscitati dai primi risultati pratici, si sono venute moltiplicando con ritmo sempre crescente, e anche oggi, nonostante la realizzazione dei primi voli umani nello spazio, il problema è sempre all'ordine del giorno, cosi che le sperimentazioni seguiteranno a svolgersi indubbiamente in avvenire, giacché $\mathbf{i}$ voli orbitali e suborbitali di astronauti sovietici e statunitensi non hanno fatto altro che ampliare i termini della ricerea senza esaurirla. Gli astronauti di queste due Nazioni pioniere sono rimasti, è vero, in condizioni di subgravità per tempi relativamente lunghi - in ogni caso molto maggiori di quelli realizzabili con qualsiasi altro mezzo -, ma tali durate non sono nemmeno lontanamente paragonabili a quelle previste per i futuri viaggi di esplorazione spaziale e planetaria, nell'ambito del nostro sistema solare e forse di là dati confini di esso, quando gli astronauti permarranno per mesi, e forse per anni, in conclizioni di subgravità e, in tale condizione, dovranno essere liberi dei loro movimenti, di attendere a mansioni molteplici, di compiere ogni sorta di atti e funzioni fisiologici, laddove gli attuali astronati sono rimasti per tutta la durata del volo vincolati al loro posto.

Anche se le previsioni più pessimistiche circa la possibilità per l'uomo di affrontare lo stato di assenza di gravità possono ormai essere scartate (i primi astronauti non hanno incontrato ostacoli di ordine 
fisiopsicologico tali da essere inibiti nello svolgimento dei compiti loro assegnati), non è tuttavia lecito affermare in piena tranquillità che la subgravita sia sotto ogni rispetto e indefinitamente innocua. Titov, l'uomo che per oltre 24 ore è rimasto in condizioni di gravità zero, ha accusato lievi disturbi come nausea e vertigrine. Tale osservazione non è stata, è vero, confermata da Glemn, il quale, eseguendo alcune prove (movimenti del capo) non riusciva a provocare in sé alcun disturbo, né trovava difficile la manovia manuale dei comandi. Comunque noi non possiamo prevedere se questi disturbi non debbano accentuarsi o cumularsi ad altri quando l'organismo umano sia, per durate indefinite, privato delle specifiche informazioni sensoriali provenientigli dal labirinto e dai vari propriocettori, anche a prescindere da conseguenze attualmente imponderabili sulla funzionalità cardiocircolatoria e di altri apparati. E per questo che taluni studiosi si sono domandati se non converrebbe mandare nello spazio individui privi per cause patologiche della funzione labirintica o addirittura labirintati di proposito!

Per aggirare questo ostacolo, vero o presunto che sia, e comunque per normalizzare il più possibile la situazione ambientale a bordo di veicoli spaziali, si è pensato di provocare una gravità artificiale mediante un'accelerazione centripeta oftenuta facendo rotare il veicolo secondo un determinato asse. Ma, come giustamente osserva Lansberg (1955), tale espediente piuttosto che evitare una serie di disturbi ne creerebbe un'altra, dato che in certe condizioni ogni movimento del capo non parallelo all'asse di rotazione provocherebbe accelemazioni e decelerazioni angolari dell'endolinfa dei canali semicircolari con la conseguenza di penose vertigini.

Comunque da queste considerazioni preliminari scaturisce la necessità di un'approfondita indagine delle reazioni di ordine fisiologico e funzionale degli organismi alla subgravita, con particolare riguardo alla funzione labirintica, di preminente importanza nello studio del problema.

$$
*^{*} *
$$

I sistemi efficaci per ottenere la subgravità, ovvero condizioni che ad essa si avvicinino almeno per taluni aspetti fisiologici, sono relativamente poco numerosi. Per mezzo di aerei supersonici, percorrenti, come si è detto, segmenti di traiettorie paraboliche, si ottengono anche parecchie decine di secondi di subgravità, fino quasi ad un minuto. Le più interessanti ricerche su soggetti umani, prima dell'avvento del volo spaziale vero e proprio, sono state condotte con questa metodica. Mediante il lancio di razzi e missili interessanti osservazioni sono state 
rondotte su animali da ricercatori americani, sovietici e francesi. Infine, mediante diversi ingegnosi sistemi, si sono ottenuti periodi di subgrarità piuttosto brevi, ma in condizioni " di laboratorio », ossia avendo a disposizione un potenziale strumentale di indagine più complesso exl efficace. Rammentiamo i sistemi a caduta, lo sfruttamento del beceheggio di un natante (von Bekh, 1959), la "torre di subgravità " (Lomonaco e coll. 1956), il cui funzionamento si fonda sul principio che un corpo lanciato da un impulso quasi istantaneo verso l'alto entra in subgravità al cessare di detto impulso e vi permane per il tempo in cui continua a salire verso l'alto, durante il tempuscolo corrispondente all'inversione del moto e nella successiva caduta fino a che non interrenga il frenamento.

Una metodica particolarmente interessante, che ha il vantaggio di non avere limitazioni di tempo, è quella dell'immersione totale del corpo umano in arqua. Data la scarsa differenza tra il peso specifico medio dell'organismo umano e quello dell'acqua, in virtù del principio di Archimede il peso dell'uomo immerso viene quasi ammullato e può essere annullato completamente usando soluzioni saline debitamente controllate. Mentre l'uniforme pressione del liquido sulla superficie corporea e il rilasciamento muscolare riducono di molto gli apporti sensoriali provenienti dall'apparato cinestetico (recettori cutanei, muscolari, ecc.). Eliminando i riferimenti visivi si riesce, con l'immersione, a sospendere tutto il complesso di elementi, che concorrono direttamente e indirettamente alla sintesi corticale che dà il senso dell'orientamento spaziale, con esclusione dei soli stimoli specifici labirintici. L'eschusione di due elementi della "triade " sensoriale che concorre all'orientamento rende la rondizione di immersione assai vicina a quella di subgravità e permette inoltre di saggiare l'importanza del senso labirintico preso da solo. Se poi applicata a soggetti privi di funzione labirintica dovrebbe generare uno stato fisiologicamente quasi sorrapponibile alla subgravità vera e propria. La tecnica dell'immersione, in mano a valenti studiosi (Margaria, 1953, 57, 58; Schock e altri) ha dato interessanti risultati cui faremo renno in seguito. Una tecnica consimile è stata impiegata da Graybiel e Clark (1961) e da Chambers e coll. (1961) con altri intendimenti: studiare riò gli effetti del rilasciamento muscolare prolungato sul rendimento psicofisico. Tali autori giungono alla previsione di una particolare sindrome definibile "astenia spaziale ".

Altre tecniche non strettamente inerenti alla produzione della subgravita, ma sempre connesse con questo problema, sono state introdotte dai ricercatori. Accenniamo alle ricerche di Arslan, di Margaria (1958) e Margaria e Gualtierotti (1959-1960). Questi due ultimi autori rilevarono 
come il grave stato di disorientamento indotto in animali (rane) col distruggere la loro funzionalità labirintica con centrifugazione violenta e intensa a poco a poco dileguava per il subentrare di una funzione vicariante da parte del senso della vista e dei vari recettori superficiali e profondi. Essi inoltre rilevavano la sensibilità clevatissima del labirinto alle accelerazioni (stimolo soglia 0,001 G) e l'esistenza di un'influenza tonica del labirinto stesso di origine spontanea e non annullata nemmeno dalla subgravità. Yeniva cosi rilevato che il labirinto deve mantenere probabilmente anche in subgravità una sua attività specifica, anche se modificata rispetto alle condizioni normali, mentre la sua funzione di orientatore sensibile alla gravità può essere sostituita da apporti sensoriali vicarianti.

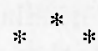

Illustriamo ora brevemente i risultati di esperimenti condotti con le tecniche descritte come efficaci a riprodurre stati di subgravità e stati a questa affini, soflermandosi in particolar morlo su quelli oftenuti dai ricercatori del Centro di Studi e Ricerche di Medicina Aeronautica e Spaziale di Roma per mezzo della " torre di subgravità".

Gli ambiti essenziali in eni si inquadrano le indagini sono, sulla traccia di von Beckh (1959): incoordinazione a disorientamento, illusioni oculoagr aviche ed effetti combinati dell'aceelerazione e della subgravità.

Nel 1952 Henry e coll. lanciavano un razzo Aerobee portante nell'ogiva topolini, parte integri e parte slabirintati, rilevandone cinematograficamente il comportamento in subgravità. Si rilevava lo stato di incoordinazione motoria e il disorientamento degli animali integri di contro al comportamento assai più normale degli animali slabirintati.

La coordinazione neuromuscolare in subgravità è stata studiata da ron Beckh (195̆4) e più tardi da Gerathewohl (1956) durante il volo parabolico. I soggetti eseguivano test grafici (tracciamento di crocette su una serie di quadretti - von Beckh - o di puntini-Gerathewohl-). Caratteristico lo stato di incoordinazione con spostamento involontario (overshoot) del tracciato verso l'alto e a destra, confermato poi da ricerche condotte con la torre di subgravità, di cui diremo in seguito.

Ballinger (1952), in studi condotti con un caccia F-80E, su soggetti legati a un giaciglio, non osservò rilevanti segni di incoordinazione e disorientamento, ma suppose che tali fenomeni si sarebbero verifiati se i soggetti fossero stati liberi di fluttuare entro la cabina, soprattutto se fossero stati privati dei riferimenti visivi. 
Tale condizione fu più tardi realizzata da diversi ricercatori. Gelathewohl (1957), nel corso di esperienze estese e sistematiche, ebbe a rilevare che circa una metà dei suoi soggetti provava un certo grado di disorientamento e di disturbo in subgravità (di rado si ebbero vertigini, nausea e fenomeni di rigurgito spontaneo), mentre per l'altra metà il fenomeno poteva addirittura avere aspetti piacevoli. L'osserrazione è interessante in quanto può porre un criterio di importanza pratica nella selezione di individui atti al volo spaziale.

Von Beckh (1959), destando improvvisamente durante la subgravità, oftenuta con volo parabolico, un soggetto in cui il sonno in volo ela stato indotto in seguito a una veglia protratta per alcuni giorni, scatenò una clamorosa condizione di disorientamento psicofisiologico e di incoordinazione motoria, soggettivamente assai penosa.

Sempre nell'ambito dell'incoordinazione motoria e del disorientamento rammentiamo le esperienze di von Beckh (1954) su una specie acquatica di tartarughe. Gli animali, portati in subgravità entro vasche, perdevano la capacità di afferrare con una mossa fulminea del collo, loro abituale, delle esche. Solo animali slabirintati da tempo mantenevano anche in subgravità la consueta rapidità e precisione. Accenniamo anche agli esperimenti di Gerathewohl e Stallings (195i) sul riflesso posturale di raddrizzamento del gatto, di provata sollecitazione labirintica, che scompare durante la subgravità.

Dalle esperienze in immersione di Margaria e coll. (1953, 1957, 1958) scaturiva la grande importanza che per l'orientamento (in questo caso riconoscimento della verticale) hanno i ricettori sensibili alla pressione e al tono muscolare, giacché i soggetti immersi bendati non erano in grado di indicare che molto approssimativamente la direzione verticale, spesso anzi incorrendo in errori grossolani. Anche tale reperto militava a favore della possibilità di funzioni vicarianti almeno in parte quella dell'apparato otolitico.

Per quanto riguarda le illusioni in subgravità bisogna riferirsi alle ricerche di Gerathewohl e Stallings (1958) e di altri, che osservarono un fenomeno, definito illusione oculo-agravica, consistente nello spostamento apparente verso l'alto di un punto luminoso, o di un'immagine postuma, osservato nell'oscurità durante lo stato di subgravità.

Altri nomi potrebbero essere citati (Strughold, Von Diringshofen, Simons, Schubert, ece.), altre ricerche potrebbero essere descritte che tralasciamo per ragioni di tempo.

Agli studi riguardanti il comportamento delle principali grandezze fisiologiche (soprattutto circolatorie e respiratorie) durante la transi- 
zione da un'accelerazione più o meno intensa allo stato di subgravità - oltre che allo studio di queste grandezze per tutto il periodo consecutivo di subgravità — vamno ascritte le principali ricerche condotte su animali lanciati in voli suborbitali e orbitali entro abitacoli spaziali, alle quali si sono aggiunte di recente le osservazioni condotte sui pionieri dello spazio, da Gagarin a Carpenter. Tale condizione di transizione, componente obbligata di qualunque volo cosmico, si è rivelata particolarmente interessinte per le sue conseguenze fisiologiche sul circolo e sul respiro. Questi esperimenti, nell'ambito della ricerca su organismi animali, comprendono i voli spaziali di cani, conigli e altri animali, realizzati da stucliosi sovietici (Kusnetzov, 1958; Gasenko et al., 1959) — tutti rammentiamo il nome di Laika, il primo animale lanciato in volo orbitale sullo Sputnik II —, di cani e di scimmie, in voli suborbitali e orbitali, operati da autori dell'U.S.A. (Burch e Gerathewohl, 1959; Graybiel e Coll., 1959), di ratti da parte di ricercatori francesi (Grandpierre e Coll., 1961).

Tutti i ricercatori sono stati concordi nel rilevare che, esclusa qualche variazione individuale e qualche fluttuazione occasionale, il comportamento fisiologico degli organismi lanciati in volo nello spazio è caratterizzato da aumento della frequenza cardiaca e respiratoria, come pure della pressione arteriosa, nella fase attiva del volo, seguito da graduale ritorno alla norma, rallentato, come vedremo, rispetto alle prove di controllo a terra, nel periodo di subgravità. Si discostano da questo schema. solo le osservazioni di Grandpierre e coll. (1961) sul ratto, ma il comportamento fisiologico di questo animale si differenzia dalla generalità degli altri mammiferi.

L'andamento della funzione cardiocircolatoria in subgravità è caratteristicamente regolare, in assenza di fenomeni abnormi di rilievo, e ciò depone favorevolmente per la sopportazione della subgravità e ha permesso a Gasenko e Kusnetzov, noti studiosi sovietici, di affermare che "le reazioni fisiologiche dell'organismo alle variazioni del campo gravitazionale non ostacoleranno la penetrazione di esseri viventi ad elevata organizzazione nello spazio cosmico ". Una previsione cosi ottimistica può essere estesa, almeno sotto il profilo della funzionalità curlio-circolatoria, e per tempi relativamente brevi, anche all'uomo. Per esempio, l'astronauta Titov (Parin, 1961) presentò una tachicardia tra 118 e 134 pulsazioni per minuto durante l'involo, sotto lo stress accelerativo, mentre la frequenza discese a 80-100 pulsazioni per minuto nella fase di subgravità, scendendo fino a 58 nel periodo di sonno. 
L'osservazione delle variazioni del comportamento di certe grandezze circolatorie e respiratorie nella fase iniziale accelerativa del volo cosmico non avrebbe importanza in questo contesto, avente per argomento la sola subgravità, tanto più perché non si rileva differenza tra questo comportamento e il comportamento fisiologico rilevato nel corso di carichi accelerativi di stessa intensità e durata indotti negli stessi animali e uomini mediante centrifuga. Essa però è stata fatta di proposito per sottolineare in seguito le differenze di reazione osservate quando all'aceelerazione non facera più seguito il ritorno alla gravità normale, come dopo prove in centrifuga, ma invece il passaggio brusco alla gravità zero. Infatti fu osservato per la prima volta sui tracciati teletrasmessi dallo Sputnik II che il periodo necessario alla frequenza cardiaca della cagna Laika per ridiscendere a valori normali era triplicato nelle condizioni di volo spaziale rispetto alle prove a terra. Von Beckh, nel 1959, fu indotto da questa osservazione, e da sue osservazioni precedenti, a riprodurre il fenomeno nell'uomo sottoponendolo a un'acelerazione, mediante una fase di volo in aereo secondo una traiettoria spirale, seguita la subgravità, mediante una traiettoria parabolica. Fu cosi osservato un protrursi dei disturbi da accelerazione durante la consecutiva subgravita. Questo autore ritenne che il fenomeno si spiegasse con un mutamento della regolazione nerrosa vegetativa in subgravità ovvero con fatti di alterazione mecanica del circolo dovuti alla riduzione del tono muscolare in subgravità che rende meno agevole la replezione e deplezione del cuore destro. Egli osservò anche, e ciò interessa la fase di rientro dal volo spaziale, che dopo subgravità la sopportazione delle accelerazioni è pure menomata. L'ulteriore conferma del fenomeno a seguito dei primi voli umani ha posto in piena luce il problema della soviapposizione degli effetti dell'accelerazione e della subgravità, problema di interesse immediato e che offre pronte possibilità di studio anche nello stato attuale della tecnica.

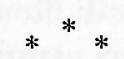

A tale genere di indagine si presta in modo particolare la "torre di subgravità ", ideata da Lomonaco e Fabris e realizzata nel 1956 presso il Centro di Studi e Ricerche di Medicina Aeronautica e Spaziale di Roma. Essa permette di ottenere brevi periodi di subgravità e gravità-zero, preceduti da un'accelerazione di $2 \div 3 \mathrm{G}$, valendosi del principio in virtì del quale un corpo lanciato da un impulso istantaneo verso l'alto viene a trovarsi in condizioni di subgravita al cessare delle forze esterne agenti su di esso. Tale stato perdura finché l'attrito con l'atmosfera, 
nella successiva caduta, o il frenamento meccanico, non sottopongano il corpo in questione a decelemazione.

Nella torre di subgravità l'impulso, utilizzato per lanciare verticalmente in alto un abitacolo, ̀̀ ottenuto per mezzo di quattro fasci di corde elastiche che, con opportuni mecranismi, vengono portati in stato di tensione e quindi rilasciati. L'abitacolo allora ì scagliato verso l'alto, con un'improvvisa accelerazione, permane alcun tempo in sub- e zero-gravità e quindi, ricadendo, è frenato dalle stesse corde elastiche e, in piccola parte, dall'attrito con l'aria, subendo una decelerazione. Si vede facilmente che un tale schema, pur con le notevoli differenze di durata totale e di multipli di $\&$ in gioco, riproduce, nella successione, le tre fasi del volo spaziale: involo, fase passiva dominata dalla subgravità, rientro.

La torre di subgravità, nella sua forma originale, era una struttura metallica, alta circa $14 \mathrm{~m}$. Il suo dispositivo di potenza, rappresentato dai quattro fasci di corde elastiche cui si è fatto cenno, permetteva il laneio di un abitacolo del peso, a vuoto, di circa $50 \mathrm{~kg}$, nel quale trovava posto un soggetto, in posizione seduta. L'accelerazione, dovuta all'impulso iniziale, era pari a $3 \mathrm{G}$; la durata del periodo di subgravità, compresa una breve fase di gravità zero, ela di 1,7 sec. Il ritorno in tensione delle corde elastiche, determinato dalla caduta dell'abitacolo, consentiva di ottenere $3-4$ cicli utili consecutivi in ciascuna prova, ognuno caratterizzato da un periodo accelerativo e da uno stato di subgravità di durata via via più breve. Le oscillazioni funali, non più utili ai fini dell'esperimento, erano smorzate da un dispositivo di frenamento; infine un dispositivo di recupero riportava a livello del suolo l'abitacolo. La durata totale della subgravita, ottenuta sommando i successivi periodi, era di cirea 4 sec. Con questo modello sono stati condotti i primi esperimenti, che illustreremo in seguito.

Più tardi (1959), sempre presso il Centro di Studi e Ricerche di Medicina Aeronautica e Spaziale di Roma, un nuovo modello di torre lat sostituito il precedente. La sua struttura perfezionata fa di esso un vero e proprio complesso subgravitazionale. La nuova torre di subgravità non differisee in principio dal modello precedente, ma, dato il suo maggiore sviluppo in altezza, e la maggiore potenza del sistema elastico propulsore, è possibile con essa ottenere periodi di subgravità di maggiore durata. Del resto è previsto (Fabris e Lomonaco, 1959) che avvalendosi di opere di ingegneria - come per esempio ponti e viadotti già esistenti, sarebbe possibile costruire dei complessi subgravitazionali di grande rendimento, capaci di ottenere periodi di subgravità della du- 
rata di molti secondi consecutivi, pur mantenendosi entro limiti di costo, sia di costruzione che di esercizio, di gran lunga inferiori a quelli di qualsiasi altro mezzo atto a ottenere veri stati di subgravità, il che potrebbe rendere i congegni fondati su questo principio strumenti di elezione per lo studio pratico in laboratorio delle condizioni di subgravità e, soprattutto, degli stadi di transizione dalla fase attiva a quella passiva del volo cosmico. D'altra parte con adatti artifizi, che sono stati applicati parzialmente all'attuale torre di subgravità, sarebbe possibile evitare un eccessivo carico accelerativo nel momento dello sgancio dellabitacolo.

L'attuale torre di subgravità, dunque, è costituita da un traliccio metallico, di strutture tubolari, appoggiato a 22 metri dal suolo sulle due ali laterali dell'edificio rhe alberga il Centro di Studi e Ricerche di Medicina Aeronautica e Spaziale di Roma. Da tale ponte metallico alcuni cavi di acciaio scendono a una base di cemento, sul suolo, intorno alla quale sono installati i dispositivi per la messa in tensione delle corde elastiche, che servono a lanciare un abitacolo verso l'alto, e quelli di sgancio, frenamento e recupero dell'abitacolo. La funzione dei avi d'acciaio tesi tra il ponte superiore e la base è essenzialmente quella di mantenere esattamente sulla verticale la direzione del moto dell'abitacolo. Questo, a differenza del primo, è una struttura chiusa di sbarre metalliche per cui il soggetto, che può indifferentemente essere disposto in posizione seduta o supina, puó essere lanciato entro l'abitacolo sia fissato con cinghie di contenzione sia libero di fluttuare durante i periodi di subgravità, ciò che effettivamente arviene, com'è documentato da riprese cinematografiche. Naturalmente, in tal caso, sono prese misure protettive per evitare l'eventuale impatto del corpo del soggetto contro le strutture dell'abitacolo stesso. Nell'abitacolo possono essere installati, a seconda delle necessità sperimentali del momento, dispositivi per la registrazione dello stato gravitazionale - accelerografo a una componente o accelerometro (l'accelerometro è utile per fissare sui singoli fotogrammi di una ripresa cinematografica ad alta velocità lo stato gravitazionale in ciascun istante) - per la ripresa cinematografica del comportamento mimico del soggetto, per l'esecuzione di radiogrammi, per la derivazione di potenziali biologici (elettrocardiogramma, nistagmogramma ecc.). Inoltre è possibile installare anche cruscotti, pamnelli o altre apparecchiature ideate per far eseguire al soggetto determinati compiti.

Senza dilungarci ulteriormente in particolari tecnici, che appesantirebbero troppo la presente trattazione, forniremo alcuni dati numerici tra i più significativi. L'abitacolo, a pieno carico (strutture proprie, apparechiature, zarorra e soggetto) ha un peso di $\mathrm{kg} 150$. Le corde 
elastiche hamno una lunghezza di 4 metri che viene portata a 12 quando, prima del lancio, vengono poste in tensione mediante appositi verricelli a mano. La spinta verticale fornita dalle corde elastiche è di $\mathrm{kg} 480$. La massima accelerazione è di 2,2 G. Come si è accennato in precerlenza, la perfetta verticalità dello spostamento è assicurata dalle corde in acciaio intomo alle quali scomono anelli metallici saldati ai lati dell'abitacolo, che impediscono eventuali sbandamenti laterali.

Seguiamo ora le fasi del lancio. Allo sganciamento segue un'acrelerazione, inizialmente di $2,2 \mathrm{G}$ - che agisce in senso testa-piedi sul soggretto seduto entro l'abitacolo - che si riduce via via fino a $1 \mathrm{G}$ in meno di 1 sec. Quando, dopo il primo secondo, l'abitacolo ha ragrgiunto l'altezar di circa 8 metri, esso si trova libero dalla propulsione elastica ed entra in subgravità $(G=0,07)$. Circa 1,2 sec più tardi esso raggiunge l'altezal massima (16 metri circa) e per un tempuscolo si trova in zero gravità assoluta. La seguente carduta, avente le caratteristiche quasi di una caduta libera, protrae lo stato di subgravità per oltre un secondo ancora, funché cioè l'abitacolo non si trova a 7 metri dal suolo. A questo punto le corde elastiche lo decelerano bruscamente, ritornando in tensione e aricandosi quindi dell'energia necessaria a determinare un successivo ciclo analogo al primo, ma naturalmente di più breve durata. Il ciclo ̀̀ durato in tutto poco meno di 4 sec, di cui oltre 2 e mezzo passati in subgravità, ossia circa un secondo di più che con il primo modello di torre. Naturalmente anche qui si ottengono $3-4$ oscillazioni utili con un solo sganciamento.

Passiamo ora a illustrare il complesso di ricerche condotte sulla subgravità dai ricereatori del Centro di Studi e Ricerche di Medicina Aeronautica e Spaziale di Roma.

Nel 1956 Lomonaco, Strollo e Fabris sottoposero a prove di coordinazione motoria 30 soggetti giovani e clinicamente sani. Nel corso di un ciclo di tre escursioni - con un periodo complessivo di subgravità di 4 sec - essi doverano eseguire un test consistente nel percuotere a ritmo naturale con un lapis un bersaglio, di una quindicina di $\mathrm{cm}$ di diametro, rappresentato da tre cerchi concentrici intersecati da due linee normali tra di loro. La prova era ripetuta per 10 volte in ciascun soggetto, dopo un congruo periodo di familiarizzazione col test, eseguito da fermo. La durata totale di ciascuna prova era di 8 sec. Furono rilevate, dal confronto con le prove di controllo eseguite dagli stessi soggetti, una notevole dispersione dei colpi su tutta la superficie del bersagrio e una frequenza alquanto aumentata. Tali risultati rispecehia vano dunque un'alterazione della coordinazione motoria verificantesi nelle condizioni 
sperimentali camatterizzate dalla transizione da un'accelerazione positiva alla subgravità. Riprese einematografiche contemporanee, e l'interrogatorio dei soggetti, rivelarono come l'esperienza potesse essere percepita come piacevole, spiacevole o indifferente a seconda dei soggetti. Tale differenza individuale di reazione riveste un notevole interesse pratico, soprattutto in vista di una futura selezione di piloti per lo spazio. Essa è confortata dalle osservazioni di altri autori (cfr. p. es. Gerathewohl, 1957). Infine è interessante rilevare che tutti o quasi i soggetti avrertirono effettivamente un senso di fluttuazione o levitazione, ancorché fossero legati al sedile, durante i periodi di subgravità.

Le osservazioni di cui sopra non permettevano però di dirimere completamente la questione se nel determinismo dei fenomeni di incoordinazione entrassero maggiormente in gioco lo stato di subgravità, o quello di accelemazione, ovvero se gli effetti di una condizione si ripercuotessero sulla seguente, data l'estrema rapidità delle alternanze. Soprattutto poi sarebbe stato importante determinare se e fino a qual punto l'apparato vestibolare dei soggetti avesse importanza nel determinismo dei fenomeni cui i soggetti emano andati incontro. A tale scopo le prove furono ripetute da Lomonaco, Scano, Strollo e Rossanigo (1957), secondo identiche modalità, su cinque individui privi di funzionalità labirintica (sordomuti). Fu molto interessante il risultato ottenuto: infatti questi soggetti non accusarono mai sensazioni fortemente sgradevoli e la loro coordinazione motoria risultò assai migliore in confronto a quella dei soggetti con labirinto normalmente funzionante. Questa era dunque la prova dell'importanza del labirinto nella genesi dei disturbi osservati.

Nel corso delle stesse esperienze si cerco anche di saggiare l'influenza che il labirinto, cosi abnormemente sollecitato, poteva avere sulla funzionalità cardiaca. Traceiati ecgrafici derivati con un caro volante durante le prove rivelarono un aumento della frequenza cardiaca, molto più spicato nei soggetti con il labirinto funzionante che in quelli privi di funzionalità labirintica. Ciò fece rilevare che accanto a una componente puramente emozionale, la tachicardia era effettivamente suscitata da stimolazioni, dirette o indirette che fossero, di origine labirintica.

Si trattava ora di approfondire le conoscenze ricavate dagli esperimenti testé descritti e di risolvere almeno alcuni dei nuovi problemi che scaturivano dalle diverse acquisizioni. In primo luogo, dato che un certo grado di disfunzione labirintica era stato messo in luce, sarebbe stato interessante rilevare quale delle due sezioni vestibolari (canali semicircolari e sistema utriculosacculare) fosse prevalentemente o esclu- 
sivamente responsabile dello scatenarsi dei disturbi. Secondariamente sarebbe stato importante rilevare le variazioni di posizione dei visceri privati momentaneamente del loro peso. Infine importava approfondire il lato psicologico, che come si è detto, è di rilerante importanza per un'eventuale selezione di sogretti per il volo cosmico.

I tre problemi furono affrontati da Lomonaco, Scano e Rossanigo (1958), con una serie di osservazioni elettronistagmografiche, radiografiche e comportamentali.

Le ricerche di nistagmografia, destinate a rispondere al primo interrogativo - individuare cioè la parte del labirinto maggiomente stimolata - furono condotte su soggetti normali, alcuni dei quali sperimentati piloti. Fu osservato che né gli stati accelerativi né la condizione di subgravità provocavano scosse nistagmiche nei soggetti, mentre, se si era indotto subito prima della prova il nistagmo mediante stimolazione termica, questo permaneva inalterato durante tutte le fasi dell'esperimento. Ciò probabilmente significava che il settore del vestibolo maggiormente interessato era il sistema utriculosacculare, come del resto era da prevedersi, mentre l'assenza di nistagmo spontaneo e l'invariabilità di quello provocato artificialmente indueevano a pensare che, anzi, lo stimolo agente sull'utricolo non veniva nemmeno trasmesso secondariamente ai canali semicircolari. Questo però non deve far pensare che i canali semicircolari non abbiano a esercitare una funzione in subgravità, perché, come vedremo in seguito, sembra che la loro integrità sia necessaria per il mantenimento dell'orientamento in subgravità.

Mediante radiogrammi eseguiti su soggetti in apnea volontaria durante la subgravità si rilevava un innalzamento, fino a $19 \mathrm{~mm}$ (in media 16) del diaframma, da attribuirsi soprattutto al cessare della trazione che gli organi ipocondriaci esercitano, a $+1 \mathrm{G}$, sul diaframma oltre che a un'espansione della massa intestinale dovuta all'elasticità naturale dei viseeri non più contrastata dalla gravità.

L'interrogatorio dei soggetti riconfermava osservazioni precedenti (senso di euforia o di malessere), e metteva in luce, quasi in ciascun soggetto, l'istintivo bisogno di irrigidirsi e di aggrapparsi a un sostegno. Due soggetti rilevarono che, eseguendo la prova a occhi chiusi, la loro sensazione era quella di compiere una serie di balzi verso l'alto, le ricadute non venendo percepite. Caratteristiche le sensazioni di galleggriamento e levitazione, che sono diventate più intense quando, in seguito alla costruzione della nuova torre, dotata di abitacolo chiuso, è stato possibile lanciare individui non vincolati al sedile e quindi liberi di effettivamente galleggiare durante la subgravità. 
Av valendosi della nuova torre, Lomonaco, Sano e Rossanigo (1960) riprendevano il problema della coordinazione percettivo-motoria e delle sue alterazioni dumante il passaggio da unaccelerazione alla sub-gravità, e clurante lo stato di subgravità. Infatti altri autori (cfr. p. es. von Beckh, 1959) avevano messo in luce, come si è detto, lo stato di incoordinazione che tali condizioni creavano nei sogretti.

Per questi esperimenti fu preparato un pannello di 30 interruttori, posti su tre file orizzontali di dieci, azionabili con leggera pressione del dito. Ciascun interuttore comandava laccensione di una lampada situata su un quadro posto alle spalle del soggetto. Tale quadro ema cinematografato (52 fotogrammi il sec) durante tutta la durata della prova. I soggetti dovevano agire sugli interruttori secondo una sequenza prestabilita, con cui si erano familiarizzati prima della prova. In prove successive tre sequenze diverse erano applicate (orizzontale, verticale e obliqua). Durante tutta la prova un accelerometro a una sola componente era in funzione. Un'esatta sincronizzazione tra lo scorrimento della pellicola e quello della registrazione accelerogratica ha permesso in seguito di riportare con precisione l'istante di accensione di ciascuma lampadina sull'accelerogramma stesso.

Fu possibile rilevare che l'attività del soggetto era discontinua, essendo praticamente sospesa nelle fasi accelerative, e concentrandosi nella fasi di subgravità. Inoltre il tempo per l'esecuzione di una sequenza era pressoché raddoppiato rispetto alle prove di controllo da fermo, mentre il numero delle imprecisioni ed errori ela accresciuto. Bisogna ricordare che i soggetti averano istruzione di eseguire la prova nel minor tempo possibile. Alcuni, che eseguirono la prova senza essere legati con la cinghia di sicurezza, presentarono un peggioramento anche piì mar(ato. E presumibile, sulla base di queste osservazioni, che lo stato di subgravità non infici gravemente l'attività percettivo-motoria, la quale invece è maggiormente influenzata dalla ipergravità. Ciò ha importanza per la predizione del comportamento di un astronauta durante le diverse fasi del volo spaziale e per la possibilità che egli abbia di eseguire attive manove di controllo sui comandi del reicolo. Dalla rilevata differenza di comportamento tra soggetti vincolati al sedile e soggetti liberi scaturisce il corollario dell'importanza, per un adeguato comportamento percettivo-motorio, di mantenere la fissità dei rapporti, mediante vincolo, tra individuo e ogetti circostanti.

Il problema, cui si è già accennato, dell'importanza di una buona funzionalità labirintica anche nelle fasi di subgravità, veniva recentemente affrontato da Caporale (1961), il quale, osservando il comporta- 
mento, nelle solite condizioni sperimentali, di colombi normali, emislabirintati, decerebrati e slabirintati, rilevava come il comportamento di un colombo privato della funzione labirintica sia molto meno coordinato di quello di un animale normale - o anche di animali emislabirintati e decerebrati - quando esso sia lasciato libero entro l'abitacolo della torre di subgravita. L'attivita dei colombi era registrata con una ripresa cinematografica. Ise esperienze eseguite in un primo tempo in gabbie aperte all'aria, sono state poi ripetute impiegando contenitori con pareti di vetro. L'identità dei risultati ha fatto rilevare come non si possa attribuire alla corrente di aria che investe l'animale un'azione disturbatrice sul comportamento. Detto comportamento consistera in uno spiegamento delle ali e movimenti di volo durante l'accelerazione, seguiti da uno stato di fluttuazione, sempre ad ali spiegate, nella subgravità, e da un atteraggio al termine di questa. Però mentre il colombo integro compie movimenti coordinati, rispondenti a riflessi che gli sono connaturati, mantiene un assetto normale ad ali spiegate, col corpo debitamente inclinato e il capo volto in alto, come nel volo normale, e, inflne, compie un esatto atterragrgio posando sui piedi, il colombo slabirintato invece si dimostra incoordinato a passivo, fluttuando in posizione inrongrua, spesso apovolta, e cadendo sul fondo della gabbia, non già atterrandovi, sul quale urta di dorso o di ala.

Ciò porterebbe alla conclusione che, negli stati di transizione dall'accelelazione alla subgravità, e durante la subgravità, una funzione integra del labirinto è necessarlia, soprattut to perché i canali semicircolari, con la loro specifica sensibilità alle variazioni delle velocità angolari, consentono il mantenimento di un congruo assetto corporeo di un organismo libero di fluttuare nell'ambiente, e quindi favoriscono la buona coordinazione e l'orientamento.

Dal complesso delle ricerche eseguite con la torre di subgravità scaturisce l'importanza del labirinto per l'orientamento e la coordinazione motoria dell'organismo umano nelle fasi del volo spaziale che comportano il passaggio dallo stato attivo a quello passivo, e durante la fase passiva presa in sé. Se da un lato le stimolazioni utrieulo-sacenlari si risolvono in un fattore forse in parte negativo, provocatore di vertigine e disturbi vari, per cui sembrerebbe auspicabile l'impiego di soggetti privi di funzione labirintica, come accennammo riferendoci in principio all'opinione di qualche autore, d'alt ra parte pare invece che una congrua funzionalità della sezione dei canali semicircolari sia necessaria per un esatto orientamento dei soggetti che affrontino la subgravità non vincolati al loro posto, ma liberi di muoversi e agire, condizione che neces- 
sariamente sarà quella degli astronanti durante i futuri viagrgi cosmici di lumghissima durata.

Si deve anche rilevare come la torre di subgravita, prescindendo anche dalle condizioni pratiche di manovabilità, sicurezza ed economia di funzionamento e costruzione, rappresenti un mezzo di laboratorio di grande e promettente portata sperimentale, la cui utilità, per risolvere numerosi problemi legati al volo spaziale e anche per proporne altri, da affrontarsi con mezzi dotati di più vaste possibilità, non può essere trascurata da chi si sforza di appianare la strada agli esploratori del cosmo.

\section{BIBLIOGRAFIA}

Armstrong II., Aerospace Medicine, William \& Wilkins, Baltimore 1960.

BaLLINGER E., Iuman esperiments in subgravity and prolonged acceleration, "J. Aviat. Merl. ", 23, 314, (1952).

Burch E.-GerathewollL S., Some observations on heart rate and cardiodynamics during weightlessness. Congr. Mond. Med. Aeron. Spaz., Roma, 1959.

Campizel B., Space Medicine. J. Marberger, Univ. Illinois Presse, 1951.

Caporale R., Comportamento di alcuni riflessi posturali nel colombo intatto, slabirintato o decerebrato, durante variazioni di accelerazione ed assenza di peso, nella torre di sub-gravità Congr. Intern. Méd. Aéron. Cosm., Paris, 1961.

Cnambers R. P. e Coll., The effects of water immersion on performance proficiency. Rep. NADC-MA-6133, U. S. Nav. Dev. Center., 22 August 1961.

Famisis L. - Lomonaco T., Congegno per creare e studiare le condizioni di subgravità nell uomo. "Congr. Mond. Med. Aeron. ", Roma, 1959.

Gasenko 0 . - Kussejzov A., Further biological investigations en rockets. Congr. Mond. Med. Aeron. Spaz. Roma, 1959.

Gauer O. - Haber H., IIan under gravity-free conditions. In "German $\Delta$ viation Medicine World War", II, (1950).

Geratnewonl S., Sensazioni soggettive durante brevi periodi di assenza di peso riferite da 16 soggetti. "Riv. ILed. Aeron. ", 20, suppl. 11. 1, 17, (1957).

GerathewomL S. e Coll., Sensomotor performance during weightlessness, eye hand coordination. "27th Ann. Meet. Aeron. Med. Ass.", Chicago 1956.

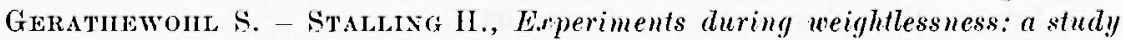
of the ooulo - agravic illusion. "J. Aviat. Med.", 29, 504, (1958).

Gerathewohl S. - Stallix: II., The labyrinthine posture refler (righting reflex) in the cat during weightlessness, "J. Aviat. Med. ", 28, 345, (1957).

Grandpierre R. e Coll., Combuicazione al Congr. Intern. Méd. Aéron. Cosm., Paris 1961.

Graymel A. e Coll., An account of experiments in which two monkeys were recovered unharmed after balistic Space hight. "Aerosp. Hed.", 30, $871,(1959)$.

Graybiel A. - Clark B., Symptoms resulting from prolonged immersion in water: the probleme of zero G asthenia. "Aerosp. Med." "32, 181, (1961). 
Gdaltinotti T. - Margaria R., Responses of the flocculo-nodular lobe of the cerebellum to minute static and dynamic excitation of the labyrinth in the curarized cat. Congr. Mond. Med. Aeron. Spaz, Roma 1959.

IIABER I1. - IIABER F., Possible methods of producing the gravity-free state for medical research. "J. Aviat. Med. ", 21, 395, (1951).

II ENin J. e Coll., Animal siudies of subgravity stales during rocket flight. "J. Aviat. Merl. ". 23, 425, (1952).

KOSNETZOV A., Some results of biological experiments on rockets and Sputnilss. Congr. Int. Méd. Aéron., Louvain 1958.

LAxsBeng .I. P., On the origin of the unpleasant sensation elicited by head movements during after-sensation. "Aeromedica Actn", 4, 67, (1955).

LAxsBsRg II. P., Further consideration on weightlesseness, artificial weight and satellite sichness. "Atti Congr. Int. Méd. Aéron. ", Louvain 1958.

Lomonaco T. - Scano A. - Rossaxigo F., Comportement de quelques données physiopsychologiques chez lhomme soumis à des variations d'accélération comprises entre 3 et 0 G. "Atti Congr. Intern. Méd. Aéron." Lonvain 1958.

Lomoxaco T. -- Scaxo A. - Rossayigo F., Comportamento di alcume funzioni percettivo motorie durante il passaggio da circa 2 a $0 \mathrm{G}$ ed infuenza dellallenamento. Esperimenti eseguiti con la torre di subgravità. "Riv. Med. Aeron. Sp. ", 23, 439, (1960).

Lowonaco T. - Scavo A. - Stroldo M. - Rossanigo F., Alcuni dati sperimentali fisiopsichici sugli effetti delle accelerazioni e della subgravità previsti nell'uomo lanciato nello spazio. "Riv. Med. Aeron. ", 20. 363, (1957).

Lomonaco T. - Strodro II. - Fanbris L., Sulla fisiopatologia durante il volo nello spazio. Comportamento della coordinazione motoria in soggetti sottoposti a valori di accelerazione variante da 3 a zero G. "Riv. Med. Acron. ", 20, suppl. 1, 76, (1957).

MaRGaRIA R., La condizione di subgravità e la soltrazione dallefjetto delle accelerazioni. "Riv. Med. Aeron.", 16, 469, (1953).

Margaria R., Le forze di accelerazione e la condizione di subgravita in volo. "Riv. Med. Aeron.", 20, 175, (1957).

IARGARIA R., Wide range acceleration investigalions in man and animals. "Riv. Med. Aeron. ", 21, 655, (1958).

Margaria R. - GUaltierotri T., Body susceptibility to high accelerations and to zero-gravity conditions. Il Congr. Internat. Aeron. Sci., Zurigo 1960.

PARIx V. V., Some results of physiological studies of man's space flight. Congr. Intern. Méd. Aéron. Cosm.. Paris 1961.

Vox BeckI H., Experimenls with animals and human subjects under suband zero-gravity conditions during the dive and parabolic Right. "J. Aviat. Med. ", 25, 235, (1954).

Vox BECKI H., Gravity changes in aircraft and ships. "J. Brit. Interplan. soc. ", 11. 73, (1956).

VON BECKI II., Flight experiments about human reaction to accelerations which are followed or preceded by the weightless state. "Aerosp. Med.", 30, 391. (1959). 